\title{
Comparison of solar radiation pressure models for BDS IGSO and MEO satellites with emphasis on improving orbit quality
}

\author{
Jing Guo ${ }^{1}$ Guo Chen ${ }^{1,2} \cdot$ Qile Zhao ${ }^{1,3} \cdot$ Jingnan Liu $^{1,3} \cdot$ Xianglin Liu $^{4}$
}

Received: 2 October 2015/ Accepted: 13 April 2016/Published online: 9 May 2016

(C) The Author(s) 2016. This article is published with open access at Springerlink.com

\begin{abstract}
In order to simplify the attitude control for inclined geosynchronous orbit (IGSO) and medium earth orbit (MEO) satellites of the BeiDou Navigation Satellite System (BDS) in eclipse seasons, two attitude modes, namely yaw-steering (YS) and orbit-normal (ON) mode are used. Significant accuracy degradation is observed for the orbits determined with the purely empirical CODE solar radiation pressure (SRP) model when these satellites switch to the ON mode. In addition, even though BDS IGSO satellites are in the YS mode, the orbits determined with the CODE SRP model show undesirable systematic errors that depend on the elevation angle $\beta$ of the sun above the satellite orbit plane and on the argument angle $\mu$ of satellite with respect to the midnight point in the orbit plane as identified from satellite laser ranging residuals. We present the yaw attitude model used for the bus of BDS IGSO and MEO satellites, and constrain the mode-switch conditions by the $\beta$ and $\mu$ angles. In order to overcome the deficiency of the purely empirical CODE SRP model for precise orbit determination (POD) of BDS IGSO and MEO satellites in the ON mode, an additional constant acceleration bias with tight constraint of
\end{abstract}

Qile Zhao

zhaoql@whu.edu.cn

1 GNSS Research Center, Wuhan University, No. 129 Luoyu Road, Wuhan 430079, China

2 School of Geodesy and Geomatics, Wuhan University, No. 129 Luoyu Road, Wuhan 430079, China

3 Collaborative Innovation Center of Earth and Space Science, Wuhan University, No. 129 Luoyu Road, Wuhan 430079, China

4 Fugro Intersite B.V., Leidschendam 2263HW, The Netherlands
$1.0 \times 10^{-10} \mathrm{~m} / \mathrm{s}^{2}$ in the along-track direction has been introduced to the CODE SRP model, and it is denoted as the C5a model. Although the orbit accuracy of IGSO and MEO satellites is significantly improved in the ON mode, the $\beta$ - and $\mu$-dependent systematic orbit errors of BDS IGSO are not reduced. Hence, with the presented yaw attitude model of the satellite bus and two assumed orientations of solar panels, the adjustable box-wing (ABW) model has been modified. Two modified ABW models are compared with the purely empirical CODE and C5a model. Based on the analysis of real data of 2014, the C5a model shows the best performance in the ON mode among the four SRP models. Although two modified ABW models show a rather worse performance for POD in the ON mode, particularly in the cross-track and radial direction, the $\beta$ - and $\mu$-dependent systematic orbit errors of BDS IGSO satellites are reduced. This provides a new insight and a possible way to improve the orbits of BDS IGSO and MEO satellites.

Keywords BDS · Yaw attitude - Solar radiation pressure Precise orbit determination - Yaw-steering (YS) mode .

Orbit-normal (ON) mode

\section{Introduction}

The BeiDou Navigation Satellite System (BDS) consists in the second phase of five geostationary orbit (GEO), five inclined geosynchronous orbit (IGSO) and four medium earth orbit (MEO) satellites (Table 1). However, C13 is currently deactivated. The constellation is unique compared with other GNSS constellations, i.e., US Global Positioning System (GPS), Russian GLObal NAvigation Satellite System (GLONASS) and European Galileo 
Table 1 Type, PRN and SVN of BDS satellites

\begin{tabular}{cccccccccccccccc}
\hline Type & GEO & \multicolumn{1}{c}{ I } & \multicolumn{1}{c}{ IGSO } & \multicolumn{11}{c}{ MEO } \\
\hline PRN & C01 & C02 & C03 & C04 & C05 & C06 & C07 & C08 & C09 & C10 & C11 & C12 & C13 & C14 \\
SVN & G01 & G06 & G03 & G04 & G05 & I01 & I02 & I03 & I04 & I05 & M03 & M04 & M05 & M06 \\
\hline
\end{tabular}

Navigation Satellite System (Galileo) formed by MEO satellites only.

For GNSS satellites, particularly for GEO and IGSO, the solar radiation pressure (SRP) is the main non-conservative orbit perturbation. The SRP acting on the satellite is difficult to model, because the resulting acceleration depends on the physical and geometrical properties of the satellite, as well as the orientation with respect to the incident radiation. Currently, several models have been proposed to model the SRP acting on the GNSS satellites and can be classified into three types: (1) Empirical models, e.g., the empirical CODE orbit model and its reduced or extend version (Beutler et al. 1994; Springer et al. 1999; Arnold et al. 2015). These models fit best the real GNSS tracking data, though they do not consider the actual physical forces acting on the satellite. (2) Analytical models based on the optical and geometrical properties of the satellite, e.g., ROCK models (Fliegel et al. 1992; Fliegel and Gallini 1996) and UCL model (Ziebart and Dare 2001). The main disadvantage of these models is that they cannot compensate accurately for the real on-orbit behavior of the satellites, e.g., due to the change or uncertainty of the a prior properties of the satellite surface or deviations from nominal attitude (Rodríguez-Solano et al. 2012). (3) Semi-analytical and semiempirical models, e.g., the adjustable boxwing model (ABW; Rodríguez-Solano et al. 2012) and GPS solar pressure model (GSPM; Bar-Sever and Kuang 2004, 2005). Such models represent intermediate approaches between analytical SRP models and empirical ones, and combine a good fit to real tracking data with a clear physical understanding of SRP.

Although the CODE SRP model was developed for use with an a priori model (Beutler et al. 1994), such as the ROCK model, a good performance can also be obtained without such a background model. Hence, the purely empirical CODE model has been used by most analysis centers of the International GNSS Service (IGS; Dow et al. 2009) for routine processing of GPS and GLONASS orbits. In addition, the purely empirical CODE model has been most widely adopted even for precise orbit determination (POD) of the newly launched GNSS satellites, due to the lack of alternative analytical SRP models. However, this model has problems with precisely representing the orbits of Galileo satellites, because satellites are markedly of cuboidal shape. With the introduction of an a priori model to the purely empirical CODE model, the deficiency has been mitigated somewhat (Montenbruck et al. 2015a).
Furthermore, the purely empirical CODE model has another deficiency regarding modeling the SRP when satellites are in eclipse seasons (Rodríguez-Solano et al. 2013). Also, the model may introduce draconitic errors into GNSS-based geodetic products (Meindl et al. 2013; Rodríguez-Solano et al. 2014). Hence, Rodríguez-Solano et al. (2012) developed an ABW model which presents the satellite as a box (satellite bus) and two wings (solar panels). By adjusting the optical properties of the satellite bus surfaces as well as solar panels, the empirical constant acceleration in $\mathrm{Y}$-axis and the solar panel lag angle, the draconitic errors are reduced (Rodríguez-Solano et al. 2014). Furthermore, the orbit quality for GPS and GLONASS in eclipse seasons could also be improved due to the fact that the ABW model accommodates intrinsically to the attitude of the satellite bus and solar panels (RodríguezSolano et al. 2013).

As previously mentioned, due to the lack of an analytical SRP model, the purely empirical CODE model has been used for BDS POD (Zhao et al. 2013; Steigenberger et al. 2013). A recent effort on SRP modeling for BDS GEO satellites has been found in Liu et al. (2016). We focus only on IGSO and MEO satellites. Satellite laser ranging (SLR) validation indicates that the orbit accuracy has reached about 10 and $5 \mathrm{~cm}$ for BDS IGSO and MEO satellites, respectively (Guo et al. 2016). However, once those satellites switch to the orbit-normal $(\mathrm{ON})$ orientation from the yaw-steering (YS) mode, the orbit accuracy degrades dramatically. This attitude-related POD issue has been analyzed in Wang et al. (2013) and Guo et al. (2013). The orbit accuracy could be improved significantly by introducing an additional empirical constant acceleration bias with a relatively tight constraint of $1.0 \times 10^{-10} \mathrm{~m} / \mathrm{s}^{2}$ in the along-track direction to the purely empirical CODE model (Zhao et al. 2013; Guo et al. 2016). However, the latter study still shows undesirable systematic errors that depend on the solar elevation angle $\beta$ above the satellite orbit plane and the argument $\mu$ of the satellite with respect to the midnight point in the orbit plane, as seen in SLR residuals of BDS IGSO satellites.

The major motivation of this study is to compare the purely empirical CODE and ABW SRP models and to analyze their impact on POD for BDS IGSO and MEO satellites in both ON and YS modes. In this study, these models are assessed and compared by orbit overlap errors and SLR validation. First, the attitude model for satellite bus and solar panels of BDS IGSO and MEO as well as the 
corresponding satellite structure are presented as prerequisites for data processing. After a short description of the POD strategy used, four solutions covering the year 2014 with different SRP models are computed for BDS IGSO and MEO satellites. These allow us to investigate the impact of SRP on orbits and seek the better approach for modeling the SRP perturbation on BDS IGSO and MEO satellites. In the last section, this study is summarized.

\section{Characteristics of BDS IGSO and MEO satellites}

As mentioned above, the SRP model developed based on the analytical approach needs to consider the details of the satellites structure, the known optical properties, the physical interaction of radiation with the satellite surfaces and the attitude of satellite bus and solar panels. These models are mainly based on information available on ground measurements provided by the satellite manufactures; however, some of them could be estimated by fitting the measurements, e.g., the attitude of satellite bus. In this section, those models will be presented for BDS IGSO and MEO satellites.

\section{Yaw attitude of satellite bus}

BDS IGSO and MEO satellites use two attitude modes, namely YS and ON modes, see Fig. 1. The orientation of these attitude modes are described in detailed by

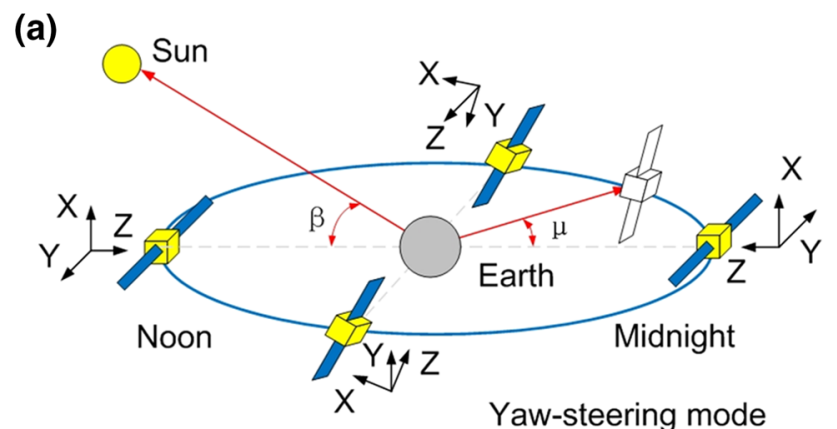

(b)

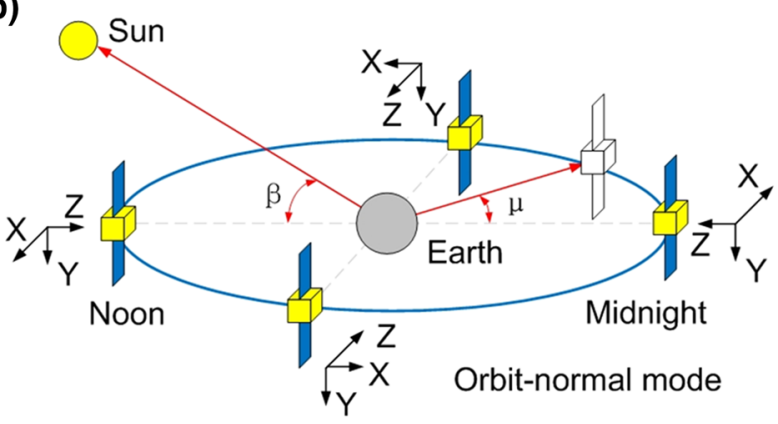

Fig. 1 BDS satellite orientation in nominal YS mode (top) and ON mode (bottom). The X-, Y- and Z-vectors indicate the axes of the satellite body-fixed frame
Montenbruck et al. (2015b). The switch of attitude control from YS to ON mode and vice versa takes place when $|\beta|$ is about $4^{\circ}$ as reported in Guo et al. (2013) and Wang et al. (2013). More recently, Guo (2014) estimated the yaw attitude profiles of BDS IGSO and MEO satellites with the reverse kinematic precise point positioning (RKPPP) approach proposed by Dilssner et al. (2011). In this approach, all relevant geodetic parameters are fixed to those estimated in POD, and the satellite clock and antenna phase center offset are estimated epoch-by-epoch using 30-s observation. The estimated horizontal antenna phase center offsets implicitly provide the spacecraft's yaw attitude. The estimates confirm the reported switch condition. However, the exact epoch of attitude switch could not be precisely determined due to the estimated errors. Hence, under the assumption that the attitude mode will switch when the yaw angle is closest to its final orientation, the exact epoch of attitude switch can be obtained as follows (Guo 2014):

The yaw attitude switches from YS mode to ON mode, and vice versa, when $\mu$ equals $90^{\circ}$, and $|\beta|$ is closest to $4^{\circ}$.

It is reasonable to make such an assumption. First, the attitude control system consumes the lowest energy when the attitude mode switches at this point. Second, but most importantly, the estimated yaw attitude indicates that the switch happens when the true satellite orientation is quite close to the target attitude as illustrated in Fig. 2, in which the nominal and estimated yaw attitude for $\mathrm{C} 06$ and $\mathrm{C} 14$ are shown. The black bars indicate the specific attitude transition epoch determined with the above-presented condition. It is worth mentioning that the condition of the orbit angle might not be well known. This problem, however, is probably not so critical since during the switch time the nominal and the $\mathrm{ON}$ attitude mode share very similar orientation.

\section{Pitch attitude of solar panels}

Besides the yaw attitude of satellite bus, the orientation of solar panels is also essential for SRP modeling, because solar panels in general are the major contributors to the large area-to-mass ratios of the satellites. In the YS mode, the surface of solar panels is perpendicular to the irradiation direction. In this case, the pitch angle of solar panels equals to the angle of sun-earth-satellite as described by Rodríguez-Solano et al. (2013). However, once the satellite switches to the ON mode, the exact attitude of solar panels is hard to be determined. In this study, two attitude modes for solar panels have been assumed in the ON mode. For the first, we still keep the surface of solar panels pointing perpendicular to the irradiation direction, and it means that the normal vector to the solar panels $\left(\vec{e}_{S P}\right)$ is parallel with the line of sight from satellite to the sun $\left(\vec{e}_{\Theta}\right)$. For the 
(a)

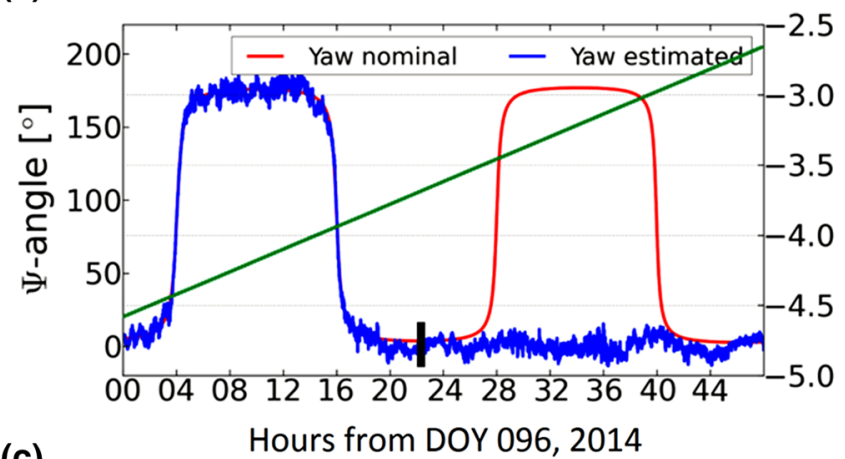

(c)

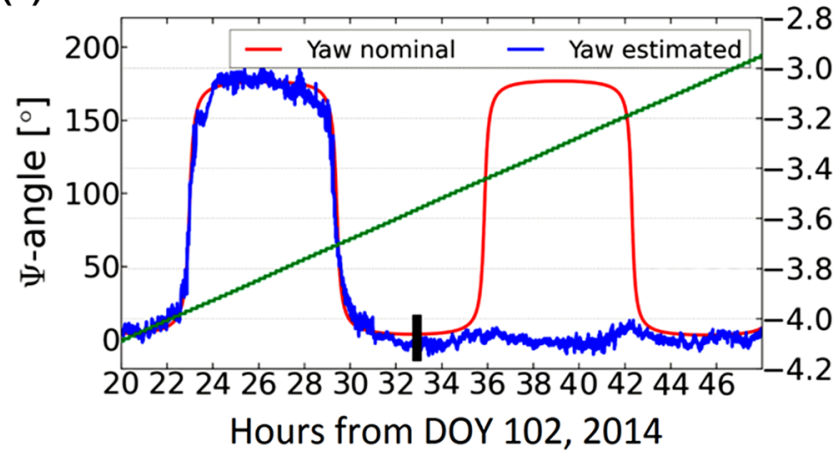

(b)

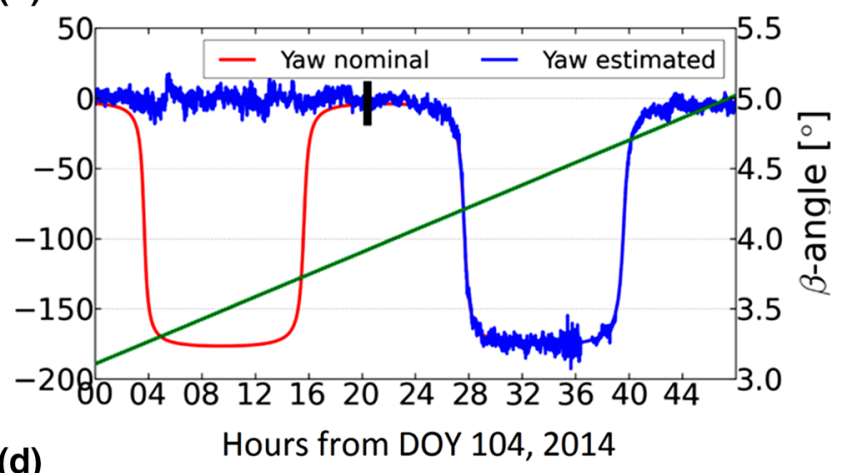

(d)

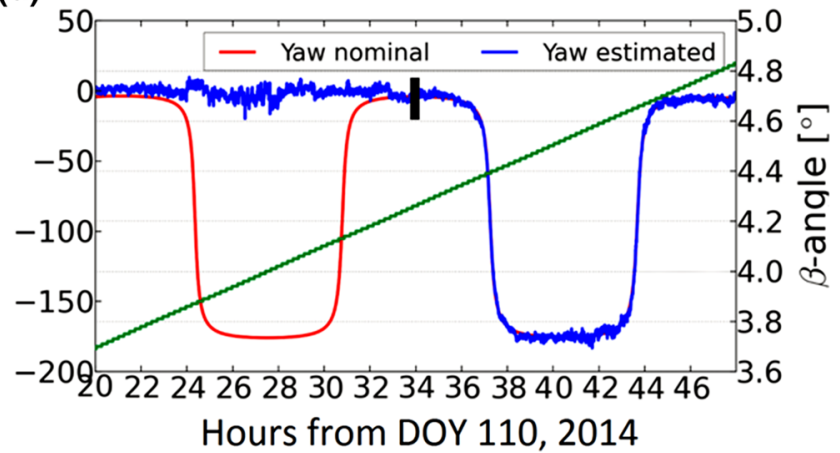

Fig. 2 Estimated (blue line) and nominal (red line) yaw angles ( $\Psi$-angle) of BDS C06 (a and b) and C14 (c and d) when satellites switch their attitude mode. The green line presents the $\beta$ angle, and the black bar indicates the attitude switch epoch derived from the attitude model

second, the normal vector to the solar panels can be obtained as follows (Rodríguez-Solano et al. 2013):

$$
\begin{array}{r}
\vec{e}_{B}=\frac{\vec{e}_{\Theta} \times \vec{e}_{S B F, Y}}{\left|\vec{e}_{\Theta} \times \vec{e}_{S B F, Y}\right|} \\
\vec{e}_{S P}=\vec{e}_{S B F, Y} \times \vec{e}_{B}
\end{array}
$$

where $\vec{e}_{S B F, Y}$ represents the Y-axis of the satellite bodyfixed frame and its orientation in the YS and ON mode could be computed according to Montenbruck et al. (2015b). In this case, the deviation of the normal direction of SPs from the irradiation direction could reach up to $4^{\circ}$ at maximum when the satellite attitude switches, and it varies with the $|\beta|$ angle. The maximum direction deviation will result in no more than $0.3 \%$ acceleration variation, but it has significant influence on the orbit modeling due to the fact that the dominant perturbation from the solar panels cannot be ignored.

\section{Satellite structure}

Besides the attitude, the analytical model needs certain a priori information of the satellite. Mandatory are realistic values of mass and dimensions, and helpful are the optical properties of the satellite surfaces. The BDS satellites of the second generation are based on the DongFangHong-3A (DFH-3A) satellite bus (http://www.cast.cn/CastCn/Show.
Table 2 Approximate values of optical and geometrical properties of BDS IGSO and MEO satellite bus and solar panels

\begin{tabular}{lclll}
\hline Panel & Area $\left(\mathrm{m}^{2}\right)$ & $\alpha$ & $\rho$ & $\delta$ \\
\hline$+\mathrm{X}$ & 3.748 & 0.350 & 0.650 & 0.0 \\
$-\mathrm{X}$ & 3.748 & 0.350 & 0.650 & 0.0 \\
$+\mathrm{Y}$ & 4.400 & 0.114 & 0.856 & 0.0 \\
$-\mathrm{Y}$ & 4.400 & 0.114 & 0.856 & 0.0 \\
$+\mathrm{Z}$ & 3.440 & 0.350 & 0.650 & 0.0 \\
$-\mathrm{Z}$ & 3.440 & 0.350 & 0.650 & 0.0 \\
Solar panels & 22.704 & 0.720 & 0.280 & 0.0 \\
\hline
\end{tabular}

asp?ArticleID=39610), which is an updated version of DFH-3 used by the first generation. The DFH-3A bus adopts a cuboidal structure. Table 2 lists the approximate optical properties related to incident radiation ( $\alpha$ absorption, $\rho$ reflection and $\delta$ diffusely scattered coefficients) and the dimensions (area of satellite bus and solar panels) of IGSO and MEO satellites as provided by the satellite manufacturer. For solar panels, the area presents the total area for the two panels. These values are used as a priori ones for the two modified ABW models in this study. Although these optical coefficients are not precise, the final orbits are slightly deteriorated, because those parameters will be fitted with real tracking measurements in the ABW model. 


\section{Processing experiments}

The data from IGS MGEX (Montenbruck et al. 2014) and BeiDou Experimental Tracking Network (BETN) in 2014 are used for data processing. The length of the POD arc is 3 days, and the POD strategy is the same as that used in Zhao et al. (2013). This study focuses on the impact of SRP models on BDS IGSO and MEO satellites, hence, the attitude model of satellite bus presented above is used directly. In total, four different SRP strategies as listed in Table 3 have been used for BDS IGSO and MEO satellites.

The C5 solution is determined with the purely empirical CODE SRP model. In this case, only five empirical parameters are estimated: three constants in the $\mathrm{D}, \mathrm{Y}$ and $\mathrm{B}$ directions (D0, Y0, and $\mathrm{B} 0$ ). and two periodic parameters representing one cycle-per-revolution variations in the $\mathrm{B}$ direction ( $\mathrm{Bc}$ and $\mathrm{Bs})$. For the $\mathrm{C} 5 \mathrm{a}$ solution, as stated before, an additional constant acceleration bias (A0) in the along-track has been introduced to the purely empirical CODE SRP model in order to reduce the deficiency of the purely empirical CODE model in the ON mode. For the other two models based on the ABW model, the original seven optical properties of the solar panels and bus surfaces, e.g., solar panel scaling factor (SPF), absorption plus diffusion of $+\mathrm{X} /+\mathrm{Z} /-\mathrm{Z}$ bus surface (corresponding parameters are indicated as $+\mathrm{XAD},+\mathrm{ZAD}$, and $-\mathrm{ZAD})$, and reflection coefficient of $+\mathrm{X} /+\mathrm{Z} /-\mathrm{Z}$ bus surface $(+\mathrm{XR}$, $+\mathrm{ZR}$, and $-\mathrm{ZR}$ ) are estimated for both ABWy and ABWo solution. In addition, the so-called Y-bias (Y0) and a parameter (SB) related to the rotation lag angle of solar panels around their rotation axis are also fitted. The reflection $(-\mathrm{XR})$ and absorption plus diffusion $(-\mathrm{XAD})$ parameters are estimated to model the accelerations caused by irradiated $-\mathrm{X}$ bus surface for ABWo solution.

The directions of the three basic axes (DYB for CODE and $\mathrm{XYZ}$ for $\mathrm{ABW}$ ) in the above SRP models used in this study have to be addressed and clarified further. The directions of D, Y and B in the C5 and C5a model can be derived from the following equations regardless of the attitude mode used,

$\vec{e}_{D}=\vec{e}_{\Theta}$

$\vec{e}_{Y}=\frac{\overrightarrow{e_{D}} \times \vec{e}_{r}}{\left|\vec{e}_{D} \times \vec{e}_{r}\right|}$

$\vec{e}_{B}=\frac{e_{D} \times e_{Y}}{\left|\vec{e}_{D} \times \vec{e}_{Y}\right|}$

where $\vec{e}_{r}$ is the unit vector pointing from the satellite to the center of earth. For the ABW model, the basic axes are in the SBF frame. Hence, it is convenient to compute the SRP perturbation acting on the satellite according to the analytical SRP model, once the attitudes of satellite bus and solar panels are known. For the ABWy and ABWo solutions, the yaw attitude of satellite bus is modeled as previously mentioned. However, following the description in the subsection on pitch attitude of solar panels attitude, we simply assume that the normal direction of solar panels is either along $\vec{e}_{\Theta}$ or computed by (1) for ABWy and ABWo, respectively. The Y-bias is modeled along $\vec{e}_{Y}$ and $\vec{e}_{S B F, Y}$ for ABWy and ABWo solutions, respectively, in order to keep it orthogonal to the normal direction of solar panels.

\section{Results and analysis}

With the previously presented attitude, POD strategy and SRP models, the impact of SRP models on the orbits of BDS IGSO and MEO satellites will be compared and analyzed based on two matrices, i.e., the orbit overlap errors and SLR validation.

\section{Orbit overlap errors}

As an internal validation of orbit accuracy, direct comparison between 48-hour overlapping portions of

Table 3 Solutions and their SRP models, attitude of solar panels used and parameters to be estimated

\begin{tabular}{|c|c|c|c|}
\hline Solutions & SRP model & Estimated parameters & $\begin{array}{l}\text { Attitude of } \\
\text { solar panels }\end{array}$ \\
\hline $\mathrm{C} 5$ & the purely empirical CODE model with 5 parameters & $\mathrm{D} 0, \mathrm{Y} 0, \mathrm{~B} 0, \mathrm{Bc}$, and $\mathrm{Bs}$ & N/A \\
\hline $\mathrm{C} 5 \mathrm{a}$ & $\begin{array}{l}\text { the purely empirical CODE model with } 5 \text { parameters } \\
\text { and an empirical constant acceleration bias in the } \\
\text { along-track component (A0) }\end{array}$ & $\mathrm{D} 0, \mathrm{Y} 0, \mathrm{~B} 0, \mathrm{Bc}, \mathrm{Bs}$, and $\mathrm{A} 0$ & N/A \\
\hline ABWy & ABW model & $\begin{array}{l}\mathrm{SPF}, \mathrm{SB}, \mathrm{Y} 0,+\mathrm{XAD},+\mathrm{ZAD}, \\
\quad-\mathrm{ZAD},+\mathrm{XR},+\mathrm{ZR} \text {, and }-\mathrm{ZR}\end{array}$ & $\vec{e}_{\Theta}$ \\
\hline ABWo & ABW model & $\begin{array}{l}\mathrm{SPF}, \mathrm{SB}, \mathrm{Y} 0,+\mathrm{XAD},+\mathrm{ZAD}, \\
\quad-\mathrm{ZAD},+\mathrm{XR},+\mathrm{ZR} \text {, and }-\mathrm{ZR}\end{array}$ & \\
\hline $\begin{array}{r}-\mathrm{XAD},-\mathrm{XR} \text { in } \\
\text { ON orientation }\end{array}$ & Equation (1) & & \\
\hline
\end{tabular}


consecutive orbit arcs is used in this study. Considering two attitude modes used by BDS IGSO and MEO satellites, only arcs having the same attitude mode are used for comparison. Those containing attitude switch epochs are removed due to significantly reduced orbit quality as shown in Guo (2014). In addition, overlapping orbits are treated as outliers and removed when the RMS values of the 3D differences are larger than 30 and $200 \mathrm{~cm}$ for arcs in the YS and ON mode, respectively. As long as one solution is treated as outlier, all four solutions are removed. Table 4 lists the numbers of all, accepted and removed POD arcs in the YS and ON mode for overlapping orbit comparison.

Figure 3 illustrates the averaged RMS values of orbit overlap errors in the along-track, cross-track and radial direction for BDS IGSO and MEO satellites in 2014, and for the four solutions listed in Table 2. The corresponding averaged 1D RMS values are listed in Table 5. The statistical results are performed separately according to the attitude modes used.

For IGSO satellites, the internal consistency of the four solutions is almost the same for POD arcs in the YS mode. However, once BDS IGSO satellites switch to the ON mode, orbit overlap errors increase no matter which kind of SRP models are used. Among the four solutions, the quality of the C5 solution degrades dramatically. The averaged 1D RMS of orbit errors increases from 6.11 to $97.85 \mathrm{~cm}$, which is dominated by larger errors in the alongtrack direction. It indicates that the mismodeled SRP perturbation is mainly along the along-track direction when satellites switch to the ON mode and that the purely empirical CODE SRP model is not suitable for POD in the ON mode. In contrast, the best internal consistency has been obtained by the C5a solution. The 1D RMS increases only from $6.64 \mathrm{~cm}$ in the YS mode to $12.02 \mathrm{~cm}$ in the ON mode (Table 5). It demonstrates that the approach may compensate mostly the deficiency of the purely empirical CODE model for POD in the ON mode. However, there are still unmodeled perturbations which need to be investigated further. The $\mathrm{ABWy}$ and $\mathrm{ABW}$ o solutions show the intermediate performance in the $\mathrm{ON}$ mode. Compared with the C5 solution, relatively larger radial errors show up in these solutions, whereas the errors in the other two components are rather lower. Although proper attitude of solar panels has been assumed for the ABWo solution, the orbit errors in each direction are larger than that of ABWy. This indicates that the assumed attitude of solar panels is incorrectly modeling the orientation of solar panels in space.
Table 4 Numbers of all, removed and accepted POD arcs for overlapping orbit comparison of BDS IGSO and MEO

Fig. 3 Averaged orbit overlap errors for BDS IGSO and MEO satellite solutions listed in Table 2 in 2014. The averages are performed separately for the YS (a, b, and $\mathbf{c})$ and ON (d, e, and f) modes. Note that different scales are for the along-track component in the ON mode

\begin{tabular}{|c|c|c|c|c|c|c|}
\hline \multirow[t]{2}{*}{ PRN } & \multirow[t]{2}{*}{ All } & \multirow{2}{*}{$\begin{array}{l}\text { Removed due to } \\
\text { attitude switch }\end{array}$} & \multicolumn{2}{|l|}{ ON } & \multicolumn{2}{|l|}{ YS } \\
\hline & & & Removed & Accepted & Removed & Accepted \\
\hline C06 & 351 & 16 & 0 & 9 & 6 & 320 \\
\hline $\mathrm{C} 07$ & 324 & 16 & 0 & 19 & 2 & 287 \\
\hline $\mathrm{C} 08$ & 354 & 18 & 0 & 11 & 1 & 324 \\
\hline C09 & 354 & 16 & 0 & 8 & 0 & 330 \\
\hline $\mathrm{C} 10$ & 350 & 16 & 0 & 19 & 0 & 315 \\
\hline $\mathrm{C} 11$ & 362 & 20 & 0 & 10 & 1 & 331 \\
\hline $\mathrm{C} 12$ & 362 & 18 & 0 & 13 & 0 & 331 \\
\hline $\mathrm{C} 14$ & 355 & 13 & 0 & 8 & 0 & 334 \\
\hline
\end{tabular}
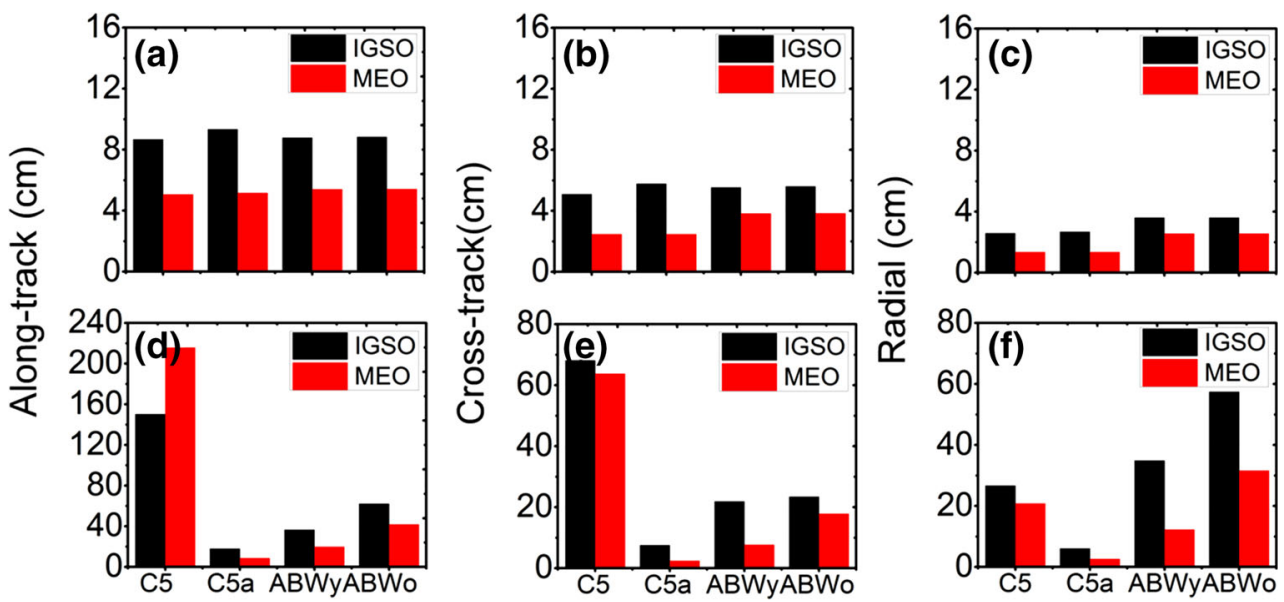
Table 5 Averaged 1D RMS of BDS IGSO and MEO orbit overlap errors for different solutions in the ON and YS mode in 2014 (unit: $\mathrm{cm})$

\begin{tabular}{llrl}
\hline Satellite type & Solutions & ON & YS \\
\hline IGSO & C5 & 97.85 & 6.11 \\
& C5a & 12.02 & 6.64 \\
& ABWy & 33.74 & 6.55 \\
\multirow{2}{*}{ MEO } & ABWo & 53.00 & 6.60 \\
& C5 & 131.08 & 3.41 \\
& C5a & 5.50 & 3.46 \\
& ABWy & 14.63 & 4.39 \\
& ABWo & 33.89 & 4.41 \\
\hline
\end{tabular}

Similar as for IGSO satellites, the C5a solution shows the best performance among the four for MOE satellites in the $\mathrm{ON}$ mode, followed by $\mathrm{ABW}$ and $\mathrm{ABW}$, whereas $\mathrm{C} 5$ is the worst, particularly in the along-track direction. However, compared with the IGSO satellites, there are some different characteristics for MEO satellites. First, the internal consistency of all MEO solutions is better than that of IGSO satellites, except the C5 solution in the ON mode. This could be contributed to a relatively better geometry strength of MEO satellites. The greatest nadir angles are about $13^{\circ}$ for MEO instead of $9^{\circ}$ for IGSO, and the relatively greater nadir angle results in better observation geometry as well as lower correlation between orbit and clock parameters. Second, compared with the similar performance achieved by the four IGSO solutions, the internal consistency of four MEO solutions in the YS mode shows a clear dependency on the basic SRP model used. Specifically, the overlapping orbit errors are about $4.4 \mathrm{~cm}$ for the $\mathrm{ABWy}$ and $\mathrm{ABW}$ o solutions, and better consistency (3.41 and $3.46 \mathrm{~cm}$ ) has been achieved by the $\mathrm{C} 5$ and $\mathrm{C} 5 \mathrm{a}$ solutions. This could be attributed to the unmodeled perturbations, e.g., earth radiation pressure. Because IGSO satellites are much further away from the earth than the MEO satellites, the impact of such unmodeled perturbations on IGSO satellites is lower than that on MEO satellites. Third, the C5 solution of MEO satellites shows more significant accuracy degradation in the along-track direction than that of IGSO satellites in the ON mode. During the time of a POD arc (72 h), MEO and IGSO satellites orbit the earth six and three times, respectively. Hence, larger mismodeled SRP perturbations accumulate in the along-track direction for MEO to degrade orbit accuracy.

In the above investigation, the similar performance of orbit solutions has been obtained with the four SRP models for POD arcs in the YS mode. However, there are still some systematic differences which are not revealed. Figure 4 illustrates the radial differences ( $\mathrm{ABWy}$ minus $\mathrm{C} 5 \mathrm{a}$ ) for $\mathrm{C} 10$ and $\mathrm{C} 11$ in the sun-fixed reference frame, which is

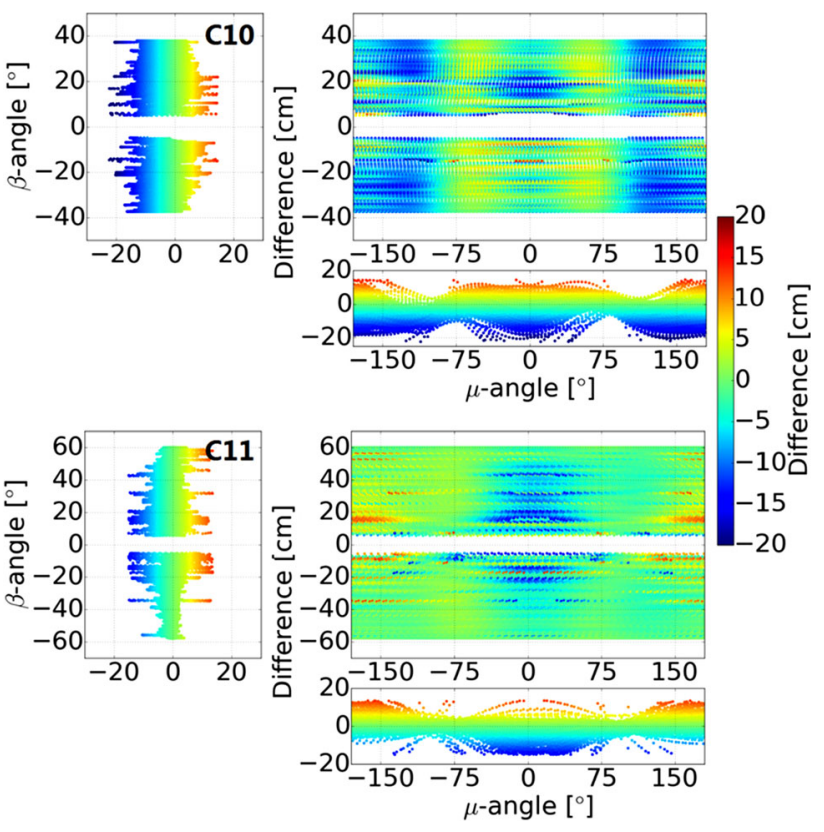

Fig. 4 Radial orbit differences (ABWy minus C5a) in the sun-fixed frame for C10 (IGSO, top panel) and C11 (MEO, bottom panel)

represented by $\beta$ and $\mu$ as shown in Fig. 1. As to the reasons why we selected radial differences for illustration: First, the radial differences can be compared with SLR residuals directly, and second, the differences in other two directions show similar pattern as that in radial. Other IGSO and MEO satellites show similar radial differences as C10 or C11. In Fig. 4, we only plot the differences for POD arcs in the YS mode. Arcs are removed once the corresponding RMS exceeds three times the average. First of all, the radial differences are satellite-type-dependent, and the averaged negative biases are about $2-3$ and $1 \mathrm{~cm}$ for BDS IGSO and MEO satellites, respectively. Second, the radial differences show an almost symmetric pattern. Although the $\beta$-dependent differences are not obvious, there is a clear $\mu$-dependency for both types of satellites. Specifically, the significant negative differences are in the $\mu$ range of $[-20$, $20],[100,150]$ and $[-150,-100]$ for $\mathrm{C} 10$, whereas they are only found in the $\mu$ range of $[-40,40]$ for $\mathrm{C} 11$. The $\mu$ variation in the radial orbit differences reflects the missing $\mu$-dependent feature of the purely empirical CODE SRP model, as this model only presents the one cycle-per-revolution signal. Hence, the resulting acceleration has a less physical meaning than that of ABWy model as shown in Fig. 5.

Figure 5 demonstrates the reconstructed SRP-induced accelerations in the along-track, cross-track, radial direction and the magnitude obtained with $\mathrm{C} 5 \mathrm{a}$ and $\mathrm{ABWy}$ for $\mathrm{C} 10$ and $\mathrm{C} 11$, respectively, when $\beta$ is abound $15^{\circ}$. In order to reconstruct the SRP accelerations, the well-estimated parameters of the ABWy and C5a SRP models which fit 

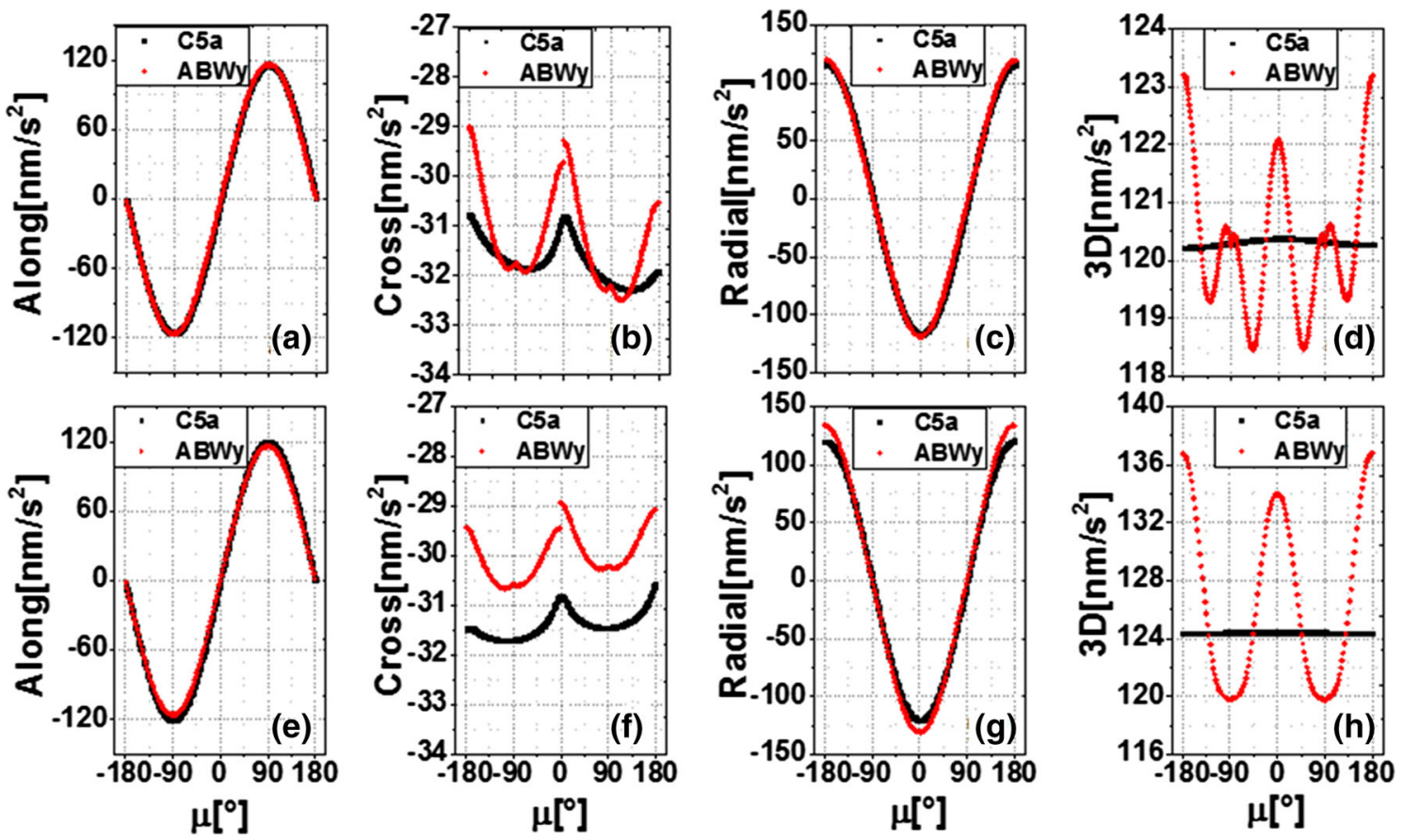

Fig. 5 Reconstructed SRP-induced accelerations for the ABWy (red) and C5a (black) model for C10 (IGSO, a-d) and C11 (MEO, e-f) when $\beta$ is about $15^{\circ}$

measurements are used. First of all, it is easy to see the larger acceleration in the cross-track as well as radial direction and the discontinue of ABWy at $\mu=0^{\circ}$, which is caused by the solar lag parameters as shown by RodríguezSolano et al. (2012) for GPS BLOCK IIA. Second, compared with $\mathrm{ABWy}$, the variation of the C5a model is much smaller and could not reflect the $\mu$-dependent features except for the one cycle-per-revolution signal. Although the differences between reconstructed accelerations of $\mathrm{C} 5 \mathrm{a}$ and $\mathrm{ABWy}$ for $\mathrm{C} 10$ and $\mathrm{C} 11$ show different behaviors, high correlation to the $\mu$-dependent radial differences for $\mathrm{C} 10$ and $\mathrm{C} 11$ can be observed, as shown in Fig. 4.

\section{SLR validation}

SLR is an optical technique providing independent validation of satellite orbits computed from GNSS observations. Basically, SLR residuals are an indicator for the radial accuracy of the GNSS orbits because the maximum incidence angle of a laser pulse to a satellite (nadir angle) is only about $9^{\circ}$ and $13^{\circ}$ for BDS IGSO and MEO satellites, respectively. Hence, the behavior of SLR residuals can be used to access the deficiency of the orbit model, particularly in the radial direction.

Although all BDS satellites are equipped with laser ranging array (LRA), only $\mathrm{C} 01, \mathrm{C} 08, \mathrm{C} 10$ and $\mathrm{C} 11$ are tracked by the International Laser Ranging Service network (ILRS; Pearlman et al. 2002). For 2014, there were 1766, 2537 and 3594 SLR normal points to C08, C10 and
C11 available for this study. Because the length of the POD arc is 3 days, only orbits in the midday are used for validation. For the SLR validation, residuals exceeding an absolute value of 50 and $300 \mathrm{~cm}$ are excluded for orbits in the YS and ON mode, respectively. Furthermore, the residuals are reedited based on the 3-sigma threshold. The numbers of SLR data used for orbit validation are listed in Table 6.

Table 7 summarizes the corresponding SLR validation results. In general, for orbits in the YS mode, the ABWy and $\mathrm{ABW}$ solutions show similar performance as expected, because the attitude of satellite bus and solar panels as well as estimated parameters are same. In addition, similar accuracy has also been achieved for C5 and C5a solutions. However, there are still some issues to be addressed. First, the orbit quality of $\mathrm{C} 11$ is better than that of $\mathrm{C} 08$ and $\mathrm{C} 10$ as indicated by SLR residuals, and the overlapping orbits of MEO satellites have also shown a better consistency than those of IGSO satellites. Obviously, this can be attributed to the better geometry condition of MEO satellites. Second, the magnitudes of negative SLR biases have become smaller by using ABWy and ABWo models instead of the C5 and C5a models. The changes are about 1.2, 1.7 and 1.0 for $\mathrm{C} 08, \mathrm{C} 10$ and $\mathrm{C} 11$, respectively, which are close to the averaged negative biases of radial differences as mentioned above. However, the orbit quality degrades by changing the C5 or C5a models with ABWy or ABWo models. The relative low quality of orbits obtained from the two models might be due to the fact that those models can only 
Table 6 Numbers of available, removed and accepted SLR normal points for BDS C08, $\mathrm{C} 10$ and $\mathrm{C} 11$ in the $\mathrm{YS}$ and $\mathrm{ON}$ modes

\begin{tabular}{|c|c|c|c|c|c|c|}
\hline \multirow[t]{2}{*}{ PRN } & \multirow[t]{2}{*}{ All NPs } & \multirow{2}{*}{$\begin{array}{l}\text { Removed due to } \\
\text { attitude switch }\end{array}$} & \multicolumn{2}{|l|}{ ON } & \multicolumn{2}{|l|}{ YS } \\
\hline & & & Removed & Accepted & Removed & Accepted \\
\hline $\mathrm{C} 08$ & 1766 & 37 & 8 & 40 & 25 & 1656 \\
\hline $\mathrm{C} 10$ & 2357 & 67 & 0 & 98 & 56 & 2136 \\
\hline $\mathrm{C} 11$ & 3594 & 162 & 6 & 80 & 64 & 3282 \\
\hline
\end{tabular}

\begin{tabular}{|c|c|c|c|c|c|c|c|}
\hline \multirow[t]{2}{*}{ Attitude } & \multirow[t]{2}{*}{ Solutions } & \multicolumn{2}{|l|}{$\mathrm{C} 08$} & \multicolumn{2}{|l|}{$\mathrm{C} 10$} & \multicolumn{2}{|l|}{$\mathrm{C} 11$} \\
\hline & & Bias & STD & Bias & STD & Bias & STD \\
\hline \multirow[t]{4}{*}{$\mathrm{ON}$} & $\mathrm{C} 5$ & 8.68 & 9.23 & 6.04 & 23.8 & -5.55 & 11.45 \\
\hline & $\mathrm{C} 5 \mathrm{a}$ & -3.11 & 9.43 & -3.42 & 13.34 & -3.10 & 2.98 \\
\hline & ABWy & -83.59 & 86.20 & -7.84 & 41.14 & 1.86 & 11.38 \\
\hline & ABWo & 68.45 & 88.36 & 21.42 & 152.23 & 19.59 & 58.74 \\
\hline \multirow[t]{4}{*}{ YS } & $\mathrm{C} 5$ & -2.84 & 4.92 & -0.47 & 5.45 & -1.42 & 3.04 \\
\hline & $\mathrm{C} 5 \mathrm{a}$ & -2.93 & 5.08 & -0.6 & 5.5 & -1.44 & 3.02 \\
\hline & ABWy & -1.75 & 5.71 & 1.08 & 5.26 & -0.31 & 4.83 \\
\hline & ABWo & -1.78 & 5.59 & 1.15 & 5.35 & -0.31 & 4.81 \\
\hline
\end{tabular}

Table 7 SLR validation for C08, C10 and C11 orbital solutions based on four SRP models in the YS and ON modes, respectively (unit: $\mathrm{cm}$ ) efficiently model the SRP, but not other perturbations, which are more easily absorbed by the purely empirical CODE model.

Once the satellites switch to the ON mode, the significant degradation of orbit quality has also been identified by SLR residuals for all solutions. In general, the ABWy and ABWo solutions for all satellites show a worse performance than the corresponding C5 and C5a solutions, except for C11 ABWy. Among these solutions, C5a still shows the best performance for both IGSO and MEO satellites. However, compared with the corresponding solutions in the YS mode, the magnitudes of biases of C5a solutions increase to $3-4 \mathrm{~cm}$, and the standard deviations (STDs) have doubled for C08 and C10. However, the STD does not change for $\mathrm{C} 11$. The greater biases and STDs are also shown in other solutions based on the C5, ABWy and ABWo models. These indicate the deficiency of SRP models in the $\mathrm{ON}$ mode. The consistent performance can also be found in orbit overlap errors in the radial component as shown in Fig. 3.

Furthermore, we analyze the SLR residuals against the $\beta$ and $\mu$ angles as we did for the radial differences. For simplification, only C5a and ABWy solutions are shown, because they show relative better performance than their counterparts, i.e., C5 and ABWo, in the ON mode. Figure 6 demonstrates the one-way SLR residuals for C5a and ABWy solutions of C08, C10 and C11. In each sub-figure, the upper left and right panels show residuals against $\beta$ only, as well as against $\beta$ and $\mu$, whereas the lower right panel shows the residuals against $\mu$ only. The upper left and lower right panels also include a moving average with a window size of 100 points (solid black line). It can be seen from Fig. 6a for the C08 C5a solution that variations of the SLR residuals show visible $\beta$ - and $\mu$-dependent behaviors. The $\beta$-dependent behavior can be approximately described as a parabola. The moving average of residuals reaches to the maximum, when $|\beta|$ is close to $4^{\circ}$ and decreases slightly to about $-1.0 \mathrm{~cm}$ as $|\beta|$ increase. For $\mu$-dependency as shown in lower right panel of Fig. 6a, the residuals are completely negative, particularly, for $\mu<-90^{\circ}$ and $\mu>90^{\circ}$ where the satellite is close to the noon point. The SLR residuals reach to positive peaks when the orbital angle is about $-50^{\circ}$ or $+50^{\circ}$. Once the SRP model is changed to ABWy (Fig. 6b), the $\beta$-dependent systematic errors have been reduced, particularly in the greater $|\beta|$ region. However, larger SLR residuals have been found at $0^{\circ} \leq|\beta|<20^{\circ}$, which do not exist for the C5a solution and are caused by relatively bad performance of orbits in the ON mode. Most importantly, the $\mu$-dependent systematic errors have been significantly reduced. The moving average line is almost straight except for some bumps caused by the larger positive SLR residuals from orbits in the ON mode. For C10 (Fig. 6c, d), similar but more visible $\mu$ dependent errors have also been found for the C5a solution. However, the $\beta$-dependent behavior is not as visible as that for $\mathrm{C} 08$, and this could be contributed to by the fact that the maximum of $|\beta|$ is about $40^{\circ}$, whereas it reaches $60^{\circ}$ for C08. With the ABWy model, the $\mu$-dependent errors have also been reduced significantly for $\mathrm{C} 10$, but there are still some larger positive SLR residuals when the satellite is near midnight $\left(50^{\circ}<\mu<-50^{\circ}\right)$. However, for $\mathrm{C} 11$ (Fig. 6e, f), no clear $\beta$ - or $\mu$-dependent systematic errors 

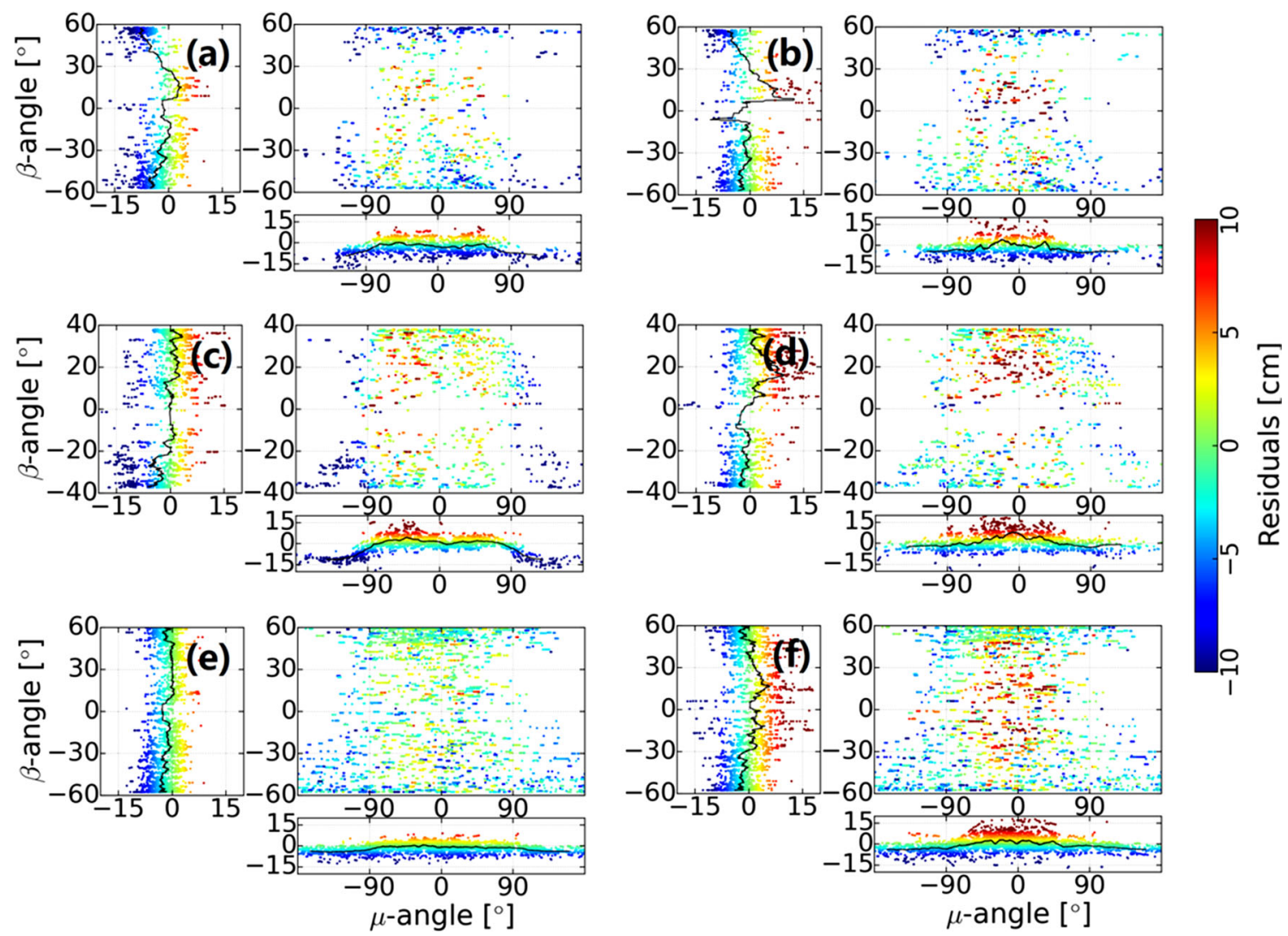

Fig. 6 One-way SLR residuals for C5a (left column) and ABWy (right column) solutions of BDS C08 (a, b), C10 (c, d), and C11 (e, f)

have been found for C5a, whereas these errors became slightly visible when the SRP model is changed to ABWy.

\section{Conclusion}

Currently, the POD for BDS satellites relies on the purely empirical CODE SRP model. However, the orbits of BDS IGSO and MEO satellites degrade significantly when satellites switch their attitude mode from the YS to ON mode. Furthermore, the SLR residuals show the $\mu$-dependent systematic errors related to the purely empirical CODE model for BDS IGSO satellites.

In this study, the CODE and ABW model have been used and modified to investigate the impact of SPR models on the orbits of BDS IGSO and MEO satellites. First, the yaw attitude model for the BDS IGSO and MEO satellite bus has been presented based on the estimation with the RKPPP approach. The geometrical and optical parameters of IGSO and MEO satellites were also presented and used as a priori information for the $\mathrm{ABW}$ model. In addition to the attitude of satellite bus, two attitude models for the orientation of the solar panels in the ON mode were proposed. The first model assumes that the normal of solar panels is along the radiation direction. The second model assumes that the solar panels are as perpendicular as possible to the sun. In order to overcome the deficiency of the purely empirical CODE SRP model for POD in the ON mode, an additional constant empirical acceleration bias with tight constraint in the along-track direction has been introduced to that model. Hence, in total, the performance of four SRP models has been analyzed and compared.

In the YS mode, in general, a similar performance of computed orbits for BDS IGSO and MEO satellites has been achieved by the purely empirical CODE and its modified model (C5 and C5a) or by the two modified ABW models (ABWy and $\mathrm{ABWo}$ ). The $\mathrm{C} 5$ and $\mathrm{C} 5 \mathrm{a}$ solutions are superior to $\mathrm{ABWy}$ and $\mathrm{ABWo}$, particularly in the crosstrack and radial direction. This could be caused by the fact that the ABW model can only efficiently model the SRP, but not the other perturbations, which are more easily absorbed by the empirical CODE model. Moreover, the orbit quality of the MEO satellites is better than that of IGSO satellites with the same SRP model due to better geometry condition. However, variations of the SLR residuals of solutions based on C5 and C5a model show visible $\beta$ - and $\mu$ - dependent errors for $\mathrm{C} 08$ and $\mathrm{C} 10$ satellites. The deficiency of the empirical CODE model could 
be reduced by exchanging $\mathrm{ABWy}$ and $\mathrm{ABW}$ model. On the other hand, the systematic errors for $\mathrm{C} 5 \mathrm{a}$ solution are nearly invisible for MEO satellites.

However, once satellites switch to the ON mode, the orbit quality degrades dramatically, particularly for the solutions based on the purely empirical CODE model (C5). It clearly indicates that the purely empirical CODE SRP model is not suitable for POD in the ON mode. Although the ABW model could adopt to the change of the satellite attitude, the two modified models still show unsatisfactory performance, possibly due to the relative larger differences between two assumed solar panel attitude models and the truth. However, different with the largest errors in the along-track direction for solutions with the CODE model, the largest differences in radial component have been obtained by the ABWy and ABWo model. Even though the C5a model shows the best performance for all validations, the performance is still not comparable with that in the YS mode, and it indicates that the SRP model still needs to be improved for POD in the ON mode. Hence, we believe that a better SRP model for BDS IGSO and MEO satellites is necessary and may be established with a combination of CODE and ABW model for BDS satellites. However, that requires further investigation without doubt.

Acknowledgments The IGS MGEX, iGMAS and ILRS are greatly acknowledged for providing the multi-GNSS and SLR tracking data. The research is partially supported by the National Natural Science Foundation of China (Grant Nos. 41404032, 41504009, 41574030), and the Fundamental Research Funds for the Central Universities of China. Finally, the authors are also grateful for the comments and remarks of two reviewers, which helped to significantly improve the manuscript.

Open Access This article is distributed under the terms of the Creative Commons Attribution 4.0 International License (http://crea tivecommons.org/licenses/by/4.0/), which permits unrestricted use, distribution, and reproduction in any medium, provided you give appropriate credit to the original author(s) and the source, provide a link to the Creative Commons license, and indicate if changes were made.

\section{References}

Arnold D, Meindl M, Beutler G, Dach R, Schaer S, Lutz S, Prange L, Sośnica K, Mervart L, Jäggi A (2015) CODE's new solar radiation pressure model for GNSS orbit determination. J Geod 89(8):775-791. doi:10.1007/s00190-015-0814-4

Bar-Sever Y, Kuang D (2004) New empirically derived solar radiation pressure model for global positioning system satellites. The Interplanetary Network Progress Report, pp 42-159

Bar-Sever Y, Kuang D (2005) New empirically derived solar radiation pressure model for global positioning system satellites during eclipse seasons. The Interplanetary Network Progress Report, pp 42-160

Beutler G, Brockmann E, Gurtner W, Hugentobler U, Mervart L, Rothacher M, Verdun A (1994) Extended orbit modeling techniques at the CODE processing center of the international
GPS service for geodynamics (IGS): theory and initial results. Manuscr Geod 19(6):367-386

Dilssner F, Springer T, Gienger G, Dow J (2011) The GLONASS-M satellite yaw-attitude model. Adv Space Res 47:160-171. doi:10. 1016/j.asr.2010.09.007

Dow J, Neilan R, Rizos C (2009) The International GNSS Service in a changing landscape of global navigation satellite systems. J Geod 83(3-4):191-198. doi:10.1007/s00190-008-0300-3

Fliegel H, Gallini T (1996) Solar force modeling of block IIR global positioning system satellites. J Spacecraft Rockets 33(6):863-866. doi: $10.2514 / 3.26851$

Fliegel H, Gallini T, Swift E (1992) Global positioning system radiation force model for geodetic applications. J Geophys Res 97(B1):559-568. doi:10.1029/91JB02564

Guo J (2014) The impacts of attitude, solar radiation and function model on precise orbit determination for GNSS satellites. PhD Dissertation (in Chinese with English abstract), GNSS Research Center, Wuhan University, Wuhan, China

Guo J, Zhao Q, Geng T, Su X, Liu J (2013) Precise orbit determination for COMPASS IGSO satellites during yaw maneuvers. In: Sun J, Jiao W, Wu H, Shi C (Eds.), Proceedings China Satellite Navigation Conference (CSNC) 2013. Vol. III. Springer 245:41-53. doi:10.1007/978-3-642-37407-4_4

Guo J, Xu X, Zhao Q, Liu J (2016) Precise orbit determination for quad-constellation satellites at Wuhan University: strategy, result validation and comparison. J Geod 90:143-159. doi:10. 1007/s00190-015-0862-9

Liu J, Gu D, Ju B, Shen Z, Lai Y, Yi D (2016) A new empirical solar radiation pressure model for BeiDou GEO satellites. Adv Space Res 57(1):234-244. doi:10.1016/j.asr.2015.10.043

Meindl M, Beutler G, Thaller D, Jäggi A, Dach R (2013) Geocenter coordinates estimated from GNSS data as viewed by perturbation theory. Adv Space Res 51(7):1047-1064. doi:10.1016/j.asr. 2012.10.026

Montenbruck O, Steigenberger P, Khachikyan R, Weber G, Langley R, Mervart L, Hugentobler U (2014) IGS-MGEX preparing the ground for multi-constellation GNSS science. Inside GNSS 9:42-49

Montenbruck O, Steigenberger P, Hugentobler U (2015a) Enhanced solar radiation pressure modeling for Galileo satellites. J Geod 89(3):283-297. doi:10.1007/s00190-014-0774-0

Montenbruck O, Schmid R, Mercier F, Steigenberger P, Noll C, Fatkulin R, Kogure S, Ganeshan AS (2015b) GNSS satellite geometry and attitude models. Adv Space Res 56:1015-1029. doi:10.1016/j.asr.2015.06.019

Pearlman M, Degnan J, Bosworth J (2002) The international laser ranging service. Adv Space Res 30(2):135-143. doi:10.1016/ S0273-1177(02)00277-6

Rodríguez-Solano C, Hugentobler U, Steigenberger P (2012) Adjustable box-wing model for solar radiation pressure impacting GPS satellites. Adv Space Res 49:1113-1128. doi:10.1016/j. asr.2012.01.016

Rodríguez-Solano C, Hugentobler U, Steigenberger P, Allende-Alba G (2013) Improving the orbits of GPS block IIA satellites during eclipse seasons. Adv Space Res 52(8):1511-1529. doi:10.1016/j. asr.2013.07.013

Rodríguez-Solano C, Hugentobler U, Steigenberger P, Blossfeld M, Fritsche M (2014) Reducing the draconitic errors in GNSS geodetic products. J Geod 88:559-574. doi:10.1007/s00190-0140704-1

Shi C, Zhao Q, Li M, Tang W, Hu Z, Lou Y, Zhang H, Niu X, Liu J (2012) Precise orbit determination of BeiDou satellites with precise positioning. Sci China Earth Sci 55(7):1079-1086. doi:10.1007/s11430-012-4446-8

Springer T, Beutler G, Rothacher M (1999) A new solar radiation pressure model for GPS satellites. GPS Solut 2(3):50-62. doi:10. 1007/PL00012757 
Steigenberger P, Hugentobler U, Hauschild A, Montenbruck O (2013) Orbit and clock analysis of compass GEO and IGSO satellites. J Geod 87:515-525. doi:10.1007/s00190-013-0625-4

Wang W, Chen G, Guo S, Song X, Zhao Q (2013) A study on the Beidou IGSO/MEO satellite orbit determination and prediction of the different yaw control mode. In: Sun J, Jiao W, Wu H, Shi C (Eds.), Proceedings China Satellite Navigation Conference (CSNC) 2013. Vol. III. Springer, pp 31-40, doi:10.1007/978-3642-37407-4 3

Zhao Q, Guo J, Li M, Qu L, Hu Z, Shi C, Liu J (2013) Initial results of precise orbit and clock determination for COMPASS navigation satellite system. J Geod 87:475-486. doi:10.1007/s00190-0130622-7

Ziebart M, Dare P (2001) Analytical solar radiation pressure modelling for GLONASS using a pixel array. J Geod 75:587-599. doi:10. 1007/s001900000136

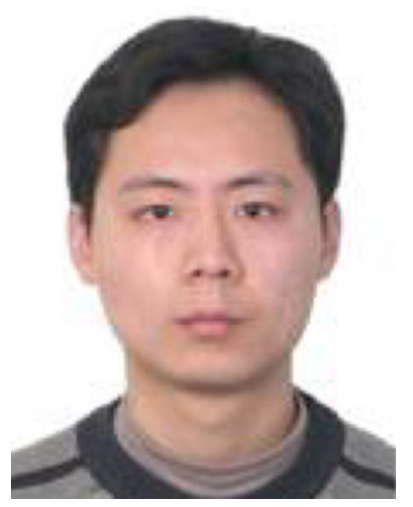

Jing Guo is a postdoctoral researcher at GNSS Research Center of Wuhan University. He received his bachelor, master and doctor degrees at Wuhan University in 2009, 2011 and 2014, respectively. Currently, he works on GNSS data processing, particularly the precise orbit determination for multiGNSS satellites, and is also responsible for the routine data processing of MGEX and iGMAS at Wuhan University.

Guo Chen is currently a $\mathrm{PhD}$ student at School of Geodesy and Geomatics of Wuhan University. He received his bachelor and master degrees at Wuhan University in 2011 and 2013. His current research mainly focuses on multi-GNSS products combination.

Qile Zhao is a professor of GNSS Research Center of Wuhan University. He received his $\mathrm{PhD}$ degree in Wuhan University in 2004. In 2006-2007, as a postdoctoral fellow, he did his postdoctoral program in DEOS, Delft University of Technology, the Netherlands. His current research interests are precise orbit determination of GNSS and low earth orbit satellites, and high-precision positioning using GPS, Galileo and BDS

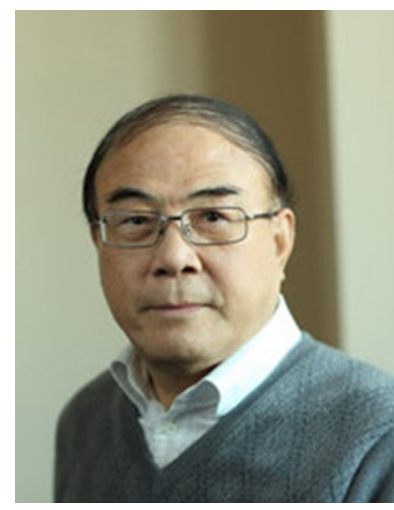

Jingnan Liu graduated from the former Wuhan College of Surveying and Mapping in 1967 and received his master's degree in 1982. He was elected Academician of the Chinese Academy of Engineering in 1999. Since 1998, he has been in charge of the National Engineering Research Center for Satellite Positioning System. He has been a member of the Science and Technology Committee, Ministry of Education of China in 1997-2009 and as an editorial board member of GPS Solutions in 1998-2000. He is currently an executive member of the council, Chinese Society for Geodesy Photogrammetry and Cartography; the editorial board member of GPS World; and the coordinator of International GPS Geodynamics Services. His current research interests are satellite precise orbit determination.

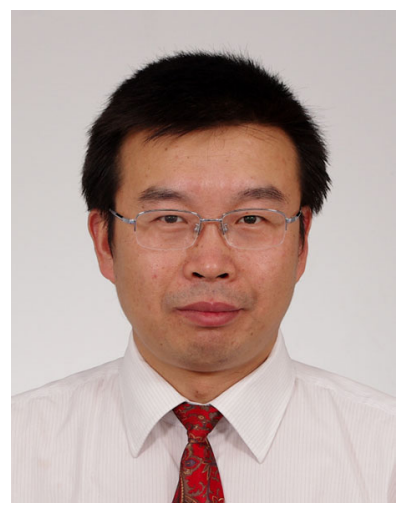

Xianglin Liu is a senior geodesist and the lead of GNSS $\mathrm{R} \& \mathrm{D}$ group at Fugro Intersite B.V., the Netherlands. $\mathrm{He}$ received his $\mathrm{PhD}$ degrees at Delft University of Technology and Wuhan University. His main research focuses on PPPRTK with multi-GNSS and multi-frequency observations.

system. 